\title{
Eventual convexity of probability constraints with elliptical distributions
}

\author{
Wim van Ackooij • Jérôme Malick
}

Received: date / Accepted: date

\begin{abstract}
Probability constraints are often employed to intuitively define safety of given decisions in optimization problems. They simply express that a given system of inequalities depending on a decision vector and a random vector is satisfied with high enough probability. It is known that, even if this system is convex in the decision vector, the associated probability constraint is not convex in general. In this paper, we show that some degree of convexity is still preserved, for the large class of elliptical random vectors, encompassing for example Gaussian or Student random vectors. More precisely, our main result establishes that, under mild assumptions, eventual convexity holds, i.e. the probability constraint is convex when the safety level is large enough. We also provide tools to compute a concrete convexity certificate from nominal problem data. Our results are illustrated on several examples, including the situation of polyhedral systems with random technology matrices and arbitrary covariance structure.
\end{abstract}

Keywords Probability constraints · Convex analysis · Elliptical distributions - Stochastic optimization

Mathematics Subject Classification (2000) MSC 90C15 · MSC 90C25 · 65K10

\section{Introduction}

1.1 Optimization with probability constraints

For a given mapping $g: \mathbb{R}^{n} \times \mathbb{R}^{m} \rightarrow \mathbb{R}^{k}$, we consider a constraint of the type:

$$
\mathbb{P}[g(x, \xi) \leq 0] \geq p,
$$

where $\xi \in \mathbb{R}^{m}$ is a random vector and $p \in(0,1)$ a user-defined safety level. Such constraints arise when a parameter $z$ of an ordinary inequality system $g(x, z) \leq 0$ is uncertain: the probability (1) gives an appropriate meaning to such an inequality system holding for sufficiently many realizations of the random vector $\xi \in \mathbb{R}^{m}$ modelling perturbations of $z$.

W. van Ackooij

EDF R\&D. OSIRIS 7, Boulevard Gaspard Monge, F-91120 Palaiseau Cedex France

E-mail: wim.van-ackooij@edf.fr

J. Malick

CNRS, LJK, F-38000 Grenoble, France

E-mail: jerome.malick@univ-grenoble-alpes.fr 
Optimization problems with such probability constraints are practically relevant as attested by numerous applications in fields as diverse as energy, telecommunications, network expansion, mineral blending, chemical engineering; see, e.g., $[1,18,35,56]$. Dealing with probability constraints is however difficult both in theory and practice. One major theoretical difficulty is that basic analytical properties of the probability function such as differentiability or convexity are not immediately derived from nominal properties of $g$. We refer the reader to $[40,42,46]$ for a comprehensive and general overview on the theory and the applications of probabilistically constrained optimization.

Although probability constraints are an intuitive and appealing model in many engineering applications, the lack of understanding of their analytical properties has limited their deployment. This has lead to an intensive development of alternatives to treat uncertainty in optimization. We refer for example to: robust optimization [3], penalty approach [12], p-efficient point based methods [9, 10,30,52], scenario approximation [6], sample average approximation [32,33,38,53], boolean reformulations [28, 29], convex approximation [37] or yet other approximations [16,22]. Common in these approaches are either the fact that $\xi$ is replaced by a random vector having a discrete and finite support (or sampled to make it so) or an (inner or outer) approximation of the feasible set to (1).

There has also been an active research directly on probability constraints, with progress in understanding their analytical properties and increasing the situations wherein they can readily be deployed. In particular, striking process have been reported on optimization algorithms adapted to probability constraints (1). We refer to the results reported in [5] (where an sequential quadratic programming (SQP) based solution methodology is suggested) and the promising bundle methods [58-60] (where convexity of the feasible set of (1) is required). Theoretical research has also brought a better understanding of the analytical properties of probability functions, including differentiability (see e.g., [54] and references therein or $[11,15,19,24,34,43,45,47-49]$ ) or generalized differentiability (see the recent papers [57] and [17]) This paper builds on this line of research: we study here the convexity properties of probabilistic functions and sets, proving in a general framework that (1) is convex for large $p \in(0,1]$.

\subsection{Convexity of probability constraints}

In this paper, we focus on a key mathematical property in view of optimization with probability constraints: convexity of (1) in a general framework. To give a taste of the type of results and assumptions that we could expect, let us start with a restricted framework. If the two following properties hold

(i) $\xi$ admits a log-concave density,

(ii) the subset of $\mathbb{R}^{n} \times \mathbb{R}^{m}$ defined by the equation $g(x, \xi) \leq 0$ is convex,

then the probability function

$$
\varphi(x)=\mathbb{P}[g(x, \xi) \leq 0]=\int 1_{\{(x, y): g(x, \xi) \leq 0\}} f(\xi) d \xi
$$

is log-concave since the integrand is so (e.g., [40, Theorem 4.2.1]). Therefore we get that the probability constraint (1) can be expressed as

$$
-\log \varphi(x) \leq-\log p
$$

establishing convexity of the constraint for all $p \in[0,1]$. This result can be further generalized stating convexity of (1) provided that $\xi$ admits a density disposing of generalized concavity properties and that $g$ is jointly quasi-convex (see, e.g., [40, section 4.6], [4], [44] or [8, Theorem 4.39] for a modern version of such results as well as [14, Proposition 4]). In these results, the restrictive assumption is the joint quasiconvexity. When $g$ fails to satisfy this property, the feasible set defined by (1) need no longer be convex for all $p \in(0,1)$ (see e.g., [23], or the forthcoming examples in Section 5). In some special cases though, convexity can still be guaranteed when $p$ is sufficiently large under some structural properties $[20,21,50,61]$. 
However existing results are restricted to a special separable structure of $g$, so that (1) involves only "lefthand side" uncertainty. To the best of our knowledge, none of the existing results cover the simple case of uncertain affine constraints under Gaussian noise

$$
A(\xi) x \leq b \quad \text { with } \quad \xi \sim N(0, I)
$$

with affine operators $A(\cdot)$ with general covariance matrix for $A(\xi)$. Recall indeed that Theorems 10.4.3, and 10.4 .5 of [40] require $A(\xi)$ to have a specially structured covariance matrix, essentially reducing the situation to the case of a single row.

In this paper, we establish a general convexity result covering the situation of (1) wherein $g$ is convex in the first argument and $\xi$ follows the so-called elliptical distribution. The class of elliptical distribution is quite large, encompassing in particular Gaussian and Student random vectors. These random vectors admit a nice "spherical-radial" decomposition that provides a convenient reformulation of probability functions. From this formulation, we show that there exists a threshold $p^{*}$ such that the set of $x$ satisfying (1) is convex for all $p>p^{*}$. Even if this threshold might be not tight, it has the interest of having an explicit analytical expression, which can be readily evaluated from concrete nominal problem data. As in the previously cited convexity results, we need in our analysis further assumptions of two types

(i) some generalized convexity properties of the law of $\xi$,

(ii) some geometrical properties of $g$.

The specifically requested properties are given in the main result, Theorem 4.1, and the subsequent discussion. Contrary to previous results though, our assumptions are general enough to cover many interesting cases, including (3). Though quite general, our assumption can be readily checked from properties of nominal problem data $g$ and $\xi$. This is in contrast with the convexity result provided in [7], relying on an abstract condition on the existence of a specific limit measure (see Definition 2 therein).

Our work is outlined as follows. Section 2.1 provides a preliminary introduction to the properties of elliptical random vectors. The results therein allow us to derive in section 2.2 a convenient reformulation of the probability function $\varphi$ of (2). This reformulation is exploited to provide useful estimates in the case that $g$ described a half-space in section 2.3. In section 3 we show how generalized concavity hidden in the nominal problem data is revealed and leads to a first concavity statement of $\varphi$ on a specific subset of $\mathbb{R}^{n}$. The main results are given in section 4 , wherein we also provide several refinements. Several illustrative examples, including the case of (3) are provided in section 5.

\subsection{Preliminaries: notation and basic assumptions}

Throughout this paper we consider a non-linear mapping $g: \mathbb{R}^{n} \times \mathbb{R}^{m} \rightarrow \mathbb{R}^{k}$ and assume that $g$ is lower semi-continuous with respect to the couple and convex with respect to the first argument. By the lower semi-continuity of $g$, the set $\mathfrak{M}(x) \subset \mathbb{R}^{m}$ defined for a given $x \in \mathbb{R}^{n}$ by

$$
\mathfrak{M}(x):=\left\{z \in \mathbb{R}^{m}: g(x, z) \leq 0\right\}
$$

is closed and consequently Borel-measurable. We can then properly define the probability function $\varphi: \mathbb{R}^{n} \rightarrow[0,1]$ by

$$
\varphi(x):=\mathbb{P}[g(x, \xi) \leq 0]=\mathbb{P}[\xi \in \mathfrak{M}(x)]
$$

Our goal is to prove that the probability constrained set

$$
M(p):=\left\{x \in \mathbb{R}^{n}: \varphi(x) \geq p\right\},
$$

is convex for large $p \in(0,1]$, under some assumptions on the function $g$ and on the random vector $\xi$. We emphasize here that we pay a special care to separate properties of $g$ and $\xi$, in our notation and the forthcoming assumptions which will be of the form (i) (ii), as above. Finally, we use the following standard notation from convex analysis: for a given set $A \subseteq \mathbb{R}^{n}, \operatorname{Co}(A)$ denotes its convex hull, cone $(A)=\mathbb{R}_{+} A$ denotes the conic hull, and $\operatorname{int} A$ refers to its interior. 


\section{Elliptical random vectors and probability functions}

In this section, we discuss the assumption that we make on the underlying randomness, and its consequences on the expressions of the probability function $\varphi$ of (5). We briefly introduce the broad class of elliptical random vectors in Section 2.1. We then study the expression of probability functions with elliptical distributions, for a general $g$ in Section 2.2 and for an affine $g$ in Section 2.3.

\subsection{Elliptical symmetrically distributed random vectors}

We say that the $m$-dimensional random vector $\xi$ is elliptically symmetrically distributed with a density parameterized by its mean $\mu$, its covariance matrix $\Sigma$ and a generator $\theta: \mathbb{R}_{+} \rightarrow \mathbb{R}_{+}$if it admits a density $f_{\xi}: \mathbb{R}^{m} \rightarrow \mathbb{R}_{+}$(with respect to the Lebesgue measure) which can be expressed by:

$$
f_{\xi}(z)=\theta\left((z-\mu)^{\top} \Sigma^{-1}(z-\mu)\right) / \sqrt{\operatorname{det} \Sigma} .
$$

The generator function $\theta: \mathbb{R}_{+} \rightarrow \mathbb{R}_{+}$may depend on the dimension, but the only requirement is that

$$
\int_{0}^{+\infty} t^{m / 2-1} \theta(t) d t<+\infty
$$

to guarantee that $f_{\xi}$ is integrable. In this paper, we use, in short, elliptical random vectors, to refer to the above definition, implicitly depending on the data $(\mu, \Sigma, \theta)$. See the textbook [13], for more information and properties on these random vectors.

The family of elliptical random vectors include many classical families: for instance, Gaussian random vectors and Student random vectors are elliptical with the respective generators

$$
\begin{aligned}
\theta^{\text {Gauss }}(t) & =\exp (-t / 2) /(2 \pi)^{m / 2} \\
\theta^{\text {Student }}(t) & =\frac{\Gamma\left(\frac{m+\nu}{2}\right)}{\Gamma\left(\frac{\nu}{2}\right)}(\pi \nu)^{-m / 2}\left(1+\frac{t}{\nu}\right)^{-\frac{m+\nu}{2}},
\end{aligned}
$$

where $\Gamma$ is the usual gamma-function. In this paper, we will illustrate our results with these two families, but our developments are valid for any elliptical random vectors. Other examples, as logistic or exponential power random vectors, are considered in the literature; see e.g. [13] and [27].

The key property for our developments is that an elliptical random vector $\xi$ admits $^{1}$ the so-called sphericalradial representation

$$
\xi=\mu+\mathcal{R} L \zeta
$$

where $L$ is a Cholesky factor of the invertible covariance matrix $\Sigma$ (a lower-triangular matrix such that $\Sigma=L L^{\top}$ ) and $\mathcal{R}$ and $\zeta$ are two independant random variables:

- $\mathcal{R}$ is a 1-dimensional random variable with support on $\mathbb{R}_{+}$(we denote its law by $\mu_{\mathcal{R}}$ )

$-\zeta$ has a uniform distribution on the $m$-dimensional euclidian sphere $\mathbb{S}^{m-1}$ (we denote its law by $\mu_{\zeta}$ ).

\footnotetext{
${ }^{1}$ For sake of completeness, let us give a short argumentation leading to it. The characteristic function of $\xi$, has the form
}

$$
\psi_{\xi}(z)=\mathbb{E}\left(\exp \left(i z^{\top} \xi\right)\right)=\exp \left(i z^{\top} \mu\right) \gamma\left(z^{\top} \Sigma z\right),
$$

for a function $\gamma: \mathbb{R} \rightarrow \mathbb{R}$, called characteristic generator. As a consequence, the characteristic function of $L^{-1}(\xi-\mu)$ satisfies $\psi_{L^{-1}(\xi-\mu)}=\gamma\left(z^{\top} z\right)$. By [13, Theorem 2.1], $L^{-1}(\xi-\mu)$ then follows a spherical distribution. It now follows from [13, Corollary to Theorem 2.2] that $L^{-1}(\xi-\mu)$ admits the representation $L^{-1}(\xi-\mu)=\mathcal{R} \zeta$, which allows us to conclude. 
Since $\xi$ has a density, so does $\mathcal{R}$ by e.g. [13, Theorem 2.9] and the density function of $\mathcal{R}$ is given by

$$
f_{\mathcal{R}}(r)=\frac{2 \pi^{\frac{m}{2}}}{\Gamma\left(\frac{m}{2}\right)} r^{m-1} \theta\left(r^{2}\right) .
$$

We refer to this density function as the radial density function. For example, when $\xi$ is Gaussian, the radial density function is

$$
f_{\mathcal{R}}^{\text {Guass }}(r)=\frac{2^{1-m / 2}}{\Gamma\left(\frac{m}{2}\right)} r^{m-1} \exp \left(-r^{2} / 2\right) .
$$

and $\mathcal{R}$ is a $\chi$ random variable with $m$ degrees of freedom. When $\xi$ is Student, $\mathcal{R}$ has a density related to $f_{m, \mu}$ the Fisher-Snedecor density with $m$ and $\nu$ degrees of freedom (see e.g. [54, (4.10)] for its explicit expression)

$$
f_{\mathcal{R}}^{\text {Student }}(r)=2 m^{-1} r f_{m, \mu}\left(m^{-1} r^{2}\right)
$$

2.2 Reformulations of the probability function

We will see in the forthcoming Theorem 2.1 that the spherical-radial decomposition of an elliptical random vector provides an alternative expression of the probability function $\varphi$ with the help of the radial distribution function of $\mathcal{R}$ and a ray function. To get to this result, we first recall that, as a consequence of (10), the probability of any Lebesgue measurable set $M \subseteq \mathbb{R}^{m}$ can be represented as

$$
\mathbb{P}(\xi \in M)=\int_{v \in \mathbb{S}^{m-1}} \mu_{\mathcal{R}}(\{r \geq 0: \mu+r L v \cap M \neq \emptyset\}) d \mu_{\zeta}(v) .
$$

We can simplify the above expression with a slight assumption on $M$. This is the content of our first result which features the ray function $\rho_{M}: \mathbb{S}^{m-1} \rightarrow\{-\infty\} \cup[0,+\infty]$ defined, for $v \in \mathbb{S}^{m-1}$, by the supremum

$$
\rho_{M}(v):=\left\{\begin{array}{l}
\sup _{t \geq 0} \quad t \\
\text { s.t. }
\end{array} \quad \mu+t L v \in M .\right.
$$

Lemma 2.1 Let $\xi \in \mathbb{R}^{m}$ be an elliptical random vector with mean $\mu$. For a Lebesgue measurable set $M \subseteq \mathbb{R}^{m}$, we have the inequality

$$
\mathbb{P}(\xi \in M) \leq \int_{v \in \mathbb{S}^{m-1}} F_{\mathcal{R}}\left(\rho_{M}(v)\right) d \mu_{\zeta}(v)
$$

where $F_{\mathcal{R}}$ is the radial distribution function of $\mathcal{R}$. Furthermore, equality holds when the set $M$ is closed and star-shaped with respect to $\mu$, which means

$$
z \in M \Longrightarrow \mu+\lambda(z-\mu) \in M \text { for all } \lambda \in[0,1]
$$

Proof We introduce the slice of $R$ defined by the intersection of $M$ with the ray directed by $L v$

$$
R(v)=\{r \geq 0: \mu+r L v \in M\} .
$$

The definition of $\rho_{M}$ yields that $R(v)$ is included in the segment of size $\rho_{M}(v)$

$$
R(v) \subseteq\left[0, \rho_{M}(v)\right]
$$

We deduce that

$$
\mu_{\mathcal{R}}(R(v)) \leq \mu_{\mathcal{R}}\left(\left[0, \rho_{M}(v)\right]\right) \leq F_{\mathcal{R}}\left(\rho_{M}(v)\right),
$$

which gives (15) from (13). Assume now that $M$ is closed and star-shaped at $\mu$. Obviously $\mu \in M$, so that $0 \in R(v)$. Note also that $\rho_{M}(v) \in R(v)$ when $\rho_{M}(v)$ is finite. Now, for any $r \in\left(0, \rho_{M}(v)\right)$, 
$\mu+r L v=\mu+\frac{r}{\rho_{M}(v)}\left(\mu+\rho_{M}(v) L v-\mu\right)$, and consequently $\mu+r L v \in M$, since $M$ is star-shaped w.r.t. $\mu$. It follows that $\left[0, \rho_{M}(v)\right] \subseteq R(v)$. Note that when $\rho_{M}(v)=\infty$, we can pick an arbitrary $\rho>0$ large enough such that $\mu+\rho L v \in M$. Arguing as above with starshapedness, we conclude that $[0, \rho] \subseteq R(v)$, which since $\rho$ was arbitrary gives $[0, \infty) \subseteq R(v)$. We have thus established equality in (15) under the additional star-shaped assumption.

Theorem 2.1 (Probability function for elliptical random vectors) Let $\xi \in \mathbb{R}^{m}$ be an elliptical random vector with mean $\mu$. Let $x \in \mathbb{R}^{n}$ be such that $g(x, \mu) \leq 0$. Assume furthermore that:

$$
g(x, \mu+\lambda(z-\mu)) \leq 0 \quad \text { for all } \lambda \in[0,1],
$$

holds for all $z$ such that $g(x, z) \leq 0$. Then the probability function $\varphi$ defined by (5) satisfies

$$
\varphi(x)=\int_{v \in \mathbb{S}^{m-1}} F_{\mathcal{R}}(\rho(x, v)) d \mu_{\zeta}(v)
$$

where the mapping $\rho: \mathbb{R}^{n} \times \mathbb{S}^{m-1} \rightarrow[-\infty,+\infty]$, called ray function, is defined from $\mathfrak{M}(x)$ in $(4)$ by

$$
\rho(x, v):=\rho_{\mathfrak{M}(x)}(v)=\left\{\begin{array}{c}
\sup _{t \geq 0} \quad t \\
s . t .
\end{array} \quad g(x, \mu+t L v) \leq 0 .\right.
$$

Proof The expression (19) comes from the definitions of $\rho_{M}$ and $\varphi$ in (14) and (5). The assumption (17) means that the set $\mathfrak{M}(x)$ is star-shaped with respect to $\mu$. We conclude by applying Lemma 2.1.

This theorem is going to be the angular stone of our developments. From the expression (18) of the probability function $\varphi$, we turn our attention in Section 3 to concavity properties of $F_{\mathcal{R}}$ and $\rho$. Here we give an illustration of (18) with a specific $\xi$. The next section studies further the case of a specific $g$, namely an affine mapping.

Example 2.1 (Student random vector) Assume that $\xi$ follows a multivariate Student distribution with mean vector $\mu$, covariance matrix $\Sigma$ and $\nu$ degrees of freedom. From (12), the radial distribution satisfies

$$
F_{\mathcal{R}}^{\text {Student }}(r)=F_{m, \mu}\left(m^{-1} r^{2}\right),
$$

where $F_{m, \nu}$ is the Fisher-Snedecor distribution with $m$ and $\nu$ degrees of freedom. Then for a general function $g$ satisfying the assumptions of Theorem 2.1. we can write

$$
\varphi(x)=\int_{v \in \mathbb{S}^{m-1}} F_{m, \nu}\left(m^{-1} \rho(x, v)^{2}\right) d \mu_{\zeta}(v)
$$

for all $x$ such that $g(x, \mu) \leq 0$.

\subsection{Case of a halfspace}

In this section, we further detail the case when $\xi$ is a general elliptical random variable, and $g$ defines a halfspace in $\mathbb{R}^{m}$. Studying this special case provides us with estimates which will be used in the sequel. We consider a single affine constraint, independent of $x$, written as

$$
g(x, z)=c^{\top} z-\gamma
$$

with $c$ such that $\left\|L^{\top} c\right\|=1$ and $\gamma \geq c^{\top} \mu$. The mapping $\rho(x, v)$ defined by (19) is the solution to the equation $c^{\top}(\mu+\rho(x, v) L v)=\gamma$ which gives

$$
\rho(x, v)=\frac{\gamma-c^{\top} \mu}{c^{\top} L v} \quad \text { when } c^{\top} L v>0
$$


and $+\infty$ elsewhere. Theorem 2.1 then gives

$$
\mathbb{P}\left[c^{\top} \xi \leq \gamma\right]=\int_{v \in \mathbb{S}^{m-1}} F_{\mathcal{R}}(\rho(x, v)) d \mu_{\zeta}(v)
$$

that we split into two parts:

$$
\begin{aligned}
\mathbb{P}\left[c^{\top} \xi \leq \gamma\right] & =\int_{c^{\top} L v>0} F_{\mathcal{R}}(\rho(x, v)) d \mu_{\zeta}(v)+\int_{c^{\top} L v \leq 0} F_{\mathcal{R}}(\rho(x, v)) d \mu_{\zeta}(v) \\
& =\int_{c^{\top} L v>0} F_{\mathcal{R}}\left(\frac{\gamma-c^{\top} \mu}{c^{\top} L v}\right) d \mu_{\zeta}(v)+\int_{c^{\top} L v \leq 0} d \mu_{\zeta}(v) \\
& =\int_{c^{\top} L v>0} F_{\mathcal{R}}\left(\frac{\gamma-c^{\top} \mu}{c^{\top} L v}\right) d \mu_{\zeta}(v)+\mu_{\zeta}\left(\left\{v \in \mathbb{S}^{m-1}: c^{\top} L v \leq 0\right\}\right) .
\end{aligned}
$$

The second term of the right-hand side raises the need to make explicit expressions of uniform measures on the sphere. In particular, we are going to use the two following results.

Lemma 2.2 (Measures on the sphere) Given $w \in \mathbb{R}^{m}$ with $\|w\|=1$ and $\delta>0$, the uniform measure of the sphere truncated at $\delta$ in the direction $w$

$$
\mathbb{S}_{\delta}^{m-1}:=\left\{v \in \mathbb{S}^{m-1}: w^{\top} v \leq \delta\right\}
$$

can be written as the ratio

$$
\mu_{\zeta}\left(\mathbb{S}_{\delta}^{m-1}\right)=\frac{\int_{\arccos \delta}^{\pi} \sin ^{m-2} \theta d \theta}{\int_{0}^{\pi} \sin ^{m-2} \theta d \theta}
$$

Proof The characterization of the uniform measure on the sphere (see e.g. [55, Lemma 4.2], [36]) states that the measure of a set $S \subset \mathbb{S}^{m-1}$ is the volume of the part of the unit ball $\mathbb{B}^{m}$ in the cone generated by $S$ divided by the volume of $\mathbb{B}^{m}$. This can be written, with $\lambda$ denoting the Lebesgue measure, as

$$
\mu_{\zeta}(S)=\lambda\left(\operatorname{cone}(S) \cap \mathbb{B}^{m}\right) / \lambda\left(\mathbb{B}^{m}\right)
$$

Let us compute these volumes for $S=\mathbb{S}_{\delta}^{m-1}$. There exists a rotation mapping for the vector $w$ of norm 1 to the last vector of the canonical basis $e_{m}=(0, \ldots, 0,1) \in \mathbb{R}^{m}$. By rotation invariance of the Lebesgue measure, we just have to compute the measure for the cases $w=e_{m}$. The volumes can be computed by integrating in spherical coordinates. The unit ball $\mathbb{B}^{m}$ can be expressed with the help of spherical coordinates as a radial coordinate $r \in[0,1]$ and angular coordinates $\theta_{1}, \ldots, \theta_{m-1}$ where the domain of $\theta_{1}$ is $[0, \pi]$ and the domain of $\theta_{i}(i=2, \ldots, m-1)$ is $[0,2 \pi]$. The set $\mathbb{S}_{\delta}^{m-1}$ also has a simple description using the same spherical coordinates with the only restriction that $\theta_{1}$ lies in $[\arccos (\delta), \pi]$. The elementary volume in spherical constraints is

$$
r^{m-1} \sin ^{m-2}\left(\theta_{1}\right) \sin ^{m-3}\left(\theta_{2}\right) \cdots \sin \left(\theta_{m-2}\right) d r d \theta_{1} \cdots d \theta_{m-1} .
$$

When forming the ratio, all the terms in the fraction simplify except the ones involving $\theta_{1}$. We end up with the announced expression by changing the notation to $\theta=\theta_{1}$.

Lemma 2.3 (Computing a given ratio) With the notation of the previous lemma, and for any given $q \in\left(0, \frac{1}{2}\right)$, there exists a unique $\delta(q)>0$ (depending on $q$ only) such that this ratio is equal to $\frac{1}{2}+q$. More precisely, $\delta(q)$ is the solution of the equation

$$
I\left(\frac{m-1}{2}, \frac{1}{2}, \sin ^{2}(\arccos (\delta))\right)=1-2 q
$$


where $I(a, b, c)$ is the normalized (incomplete) Beta function

$$
I(a, b, c)=\frac{\int_{0}^{c} t^{a-1}(1-t)^{b-1} d t}{\int_{0}^{1} t^{a-1}(1-t)^{b-1} d t}
$$

which is a standard function admitting efficient high-accuracy calculators. Hence we can compute $\delta(q)$ quickly by binary search.

Proof The ratio (21) is strictly increasing from 0 at $\delta=-1$ to $1 \delta=1$, and it is equal to $1 / 2$ at $\delta=0$. This proves existence and uniqueness of $\delta(q)>0$ such that the ratio equals $q+\frac{1}{2}$ for a $q \in\left(0, \frac{1}{2}\right)$. Equation (22) then comes from calculation using the standard Beta function $\mathfrak{B}(a, b)$ which is the denominator of the fraction. Note in particular that by a change of variables in the integral, we get

$$
\mathfrak{B}\left(\frac{m-1}{2}, \frac{1}{2}\right)=2 \int_{0}^{\pi / 2} \sin ^{m-2} \theta d \theta
$$

The rest follows from straightforward calculations.

Theorem 2.2 Let $\xi$ be an elliptical random vector. Then, for any $q \in\left(0, \frac{1}{2}\right)$, there exists a unique $\delta(q)>0$ such that for any $c \in \mathbb{R}^{m}$ such that $\left\|L^{\top} c\right\|=1$ and $c^{\top} \mu \leq \gamma$ we have

$$
\begin{aligned}
\mathbb{P}\left[c^{\top} \xi \leq \gamma\right] & =\int_{c^{\top} L v>0} F_{\mathcal{R}}\left(\frac{\gamma-c^{\top} \mu}{c^{\top} L v}\right) d \mu_{\zeta}(v)+\frac{1}{2} \\
& \leq\left(\frac{1}{2}-q\right) F_{\mathcal{R}}\left(\frac{\gamma-c^{\top} \mu}{\delta(q)}\right)+\left(q+\frac{1}{2}\right) .
\end{aligned}
$$

Proof The equality (23) comes directly from the preceding developments together with the fact that the uniform measure of the half-sphere is equal to $1 / 2$ (by the expression of Lemma 2.2). In order to establish (24), we now refine the argument. We fix $\delta>0$, and split the integral (20) over $v \in \mathbb{S}^{m-1}$ into the two sets defined by $c^{\top} L v \geq \delta$ and $\delta>c^{\top} L v$. Since the cumulative distribution is increasing, we can bound

$$
F_{\mathcal{R}}\left(\frac{\gamma-c^{\top} \mu}{c^{\top} L v}\right) \leq F_{\mathcal{R}}\left(\frac{\gamma-c^{\top} \mu}{\delta}\right) \quad \text { when } c^{\top} L v \geq \delta .
$$

We simply bound it by 1 when $\delta>c^{\top} L v$. Then we get

$$
\mathbb{P}\left[c^{\top} \xi \leq \gamma\right] \leq F_{\mathcal{R}}\left(\frac{\gamma-c^{\top} \mu}{\delta}\right) \int_{c^{\top} L v \geq \delta} d \mu_{\zeta}(v)+\int_{\delta>c^{\top} L v} d \mu_{\zeta}(v) .
$$

The two measures appearing in this bound can be written with the help of $\mu_{\zeta}\left(\mathbb{S}_{\delta}^{m-1}\right)$ of Lemma 2.2, which yields

$$
\mathbb{P}\left[c^{\top} \xi \leq \gamma\right] \leq F_{\mathcal{R}}\left(\frac{\gamma-c^{\top} \mu}{\delta}\right)\left(1-\mu_{\zeta}\left(\mathbb{S}_{\delta}^{m-1}\right)\right)+\mu_{\zeta}\left(\mathbb{S}_{\delta}^{m-1}\right) .
$$

For a given $q \in(0,1 / 2)$, we pick the $\delta=\delta(q)$ given in Lemma 2.3 to obtain (24).

We finish this section with a simple corollary of the above result that will be used in the proof of the main result.

Corollary 2.1 Let a convex set $M \subseteq \mathbb{R}^{m}$ and an elliptical random vector $\xi$ be given. If $\mathbb{P}[\xi \in M]>\frac{1}{2}$ holds, then $\mu$ lies in the interior of $M$. 
Proof Let us prove the inverse implication, namely: if $\mu \notin \operatorname{int} M$, then $\mathbb{P}[\xi \in M] \leq \frac{1}{2}$. When $\mu \notin \operatorname{int} M$, we can separate $\mu$ and $M$ : there exists a scalar $\gamma$ and a vector $c$ (w.l.o.g., we may assume $\left\|L^{\top} c\right\|=1$ ) such that

$$
c^{\top} \mu \leq \gamma \quad \text { and } \quad M \subseteq\left\{z \in \mathbb{R}^{m}: c^{\top} z \geq \gamma\right\} .
$$

In particular $c^{\top} \mu \leq \gamma$. We deduce that $\mathbb{P}[\xi \in M] \leq 1-\mathbb{P}\left[c^{\top} \xi \leq \gamma\right]$. We can conclude, since (23) guarantees that $\mathbb{P}\left[c^{\top} \xi \leq \gamma\right] \geq 1 / 2$.

\section{Convexity properties of $F_{\mathcal{R}}, \rho$, and $\varphi$}

\subsection{Concavity revealed in radial distribution functions}

In this section, we study the generalized concavity properties present in many radial distribution functions of elliptical random vectors. To this end, we introduce a notion, that we call $\alpha$-revealed-concavity.

Definition 3.1 ( $\alpha$-revealed-concavity) We say that $f: \mathbb{R} \rightarrow \mathbb{R} \cup\{+\infty\}$ is $\alpha$-revealed-concave for a given $\alpha \in(-\infty, 1]$ if there exists a $t(\alpha) \in \mathbb{R}$ such that one of the three following conditions holds:

$-\alpha<0$ and $t \mapsto f\left(t^{\frac{1}{\alpha}}\right)$ is concave on $(0, t(\alpha)]$

$-\alpha=0$ and $t \mapsto f(\exp (t))$ is concave on $[t(\alpha), \infty)$

$-\alpha>0$ and $t \mapsto f\left(t^{\frac{1}{\alpha}}\right)$ is concave on $[t(\alpha), \infty)$.

Although this notion looks restrictive, it is actually rather weak and generally satisfied for the functions of interest, as our next lemma and examples show (see e.g., Table 1 below).

Lemma 3.1 Let $\theta: \mathbb{R}_{+} \rightarrow \mathbb{R}_{+}$be a differentiable generator of an elliptical distribution. Then, for a given $\alpha \in \mathbb{R}$, its associated radial distribution $F_{\mathcal{R}}$ is $\alpha$-revealed-concave if one of the two inequalities

$$
\begin{array}{lll}
\alpha \neq 0 & \text { and } & (m-\alpha) \theta\left(t^{\frac{2}{\alpha}}\right)+2 t^{\frac{2}{\alpha}} \theta^{\prime}\left(t^{\frac{2}{\alpha}}\right) \leq 0 \\
\alpha=0 & \text { and } & m \theta\left(e^{2 t}\right)+2 e^{2 t} \theta^{\prime}\left(e^{2 t}\right) \leq 0 .
\end{array}
$$

holds. In this case, $t(\alpha)$ is the smallest solution to the equation corresponding the above inequality taken as an equality.

Proof Let us prove the result for the case $\alpha<0$ as the two other cases follow almost identically. Denote $h(t)=F_{\mathcal{R}}\left(t^{\frac{1}{\alpha}}\right)$ and take the first and second derivative of $h$

$$
\begin{aligned}
h^{\prime}(t) & =f_{\mathcal{R}}\left(t^{\frac{1}{\alpha}}\right) \frac{1}{\alpha} t^{\frac{1-\alpha}{\alpha}}=\frac{2 \pi^{\frac{m}{2}}}{\Gamma\left(\frac{m}{2}\right)} \frac{1}{\alpha} t^{\frac{m-\alpha}{\alpha}} \theta\left(t^{\frac{2}{\alpha}}\right), \\
h^{\prime \prime}(t) & =\frac{2 \pi^{\frac{m}{2}}}{\Gamma\left(\frac{m}{2}\right)} \frac{1}{\alpha^{2}} t^{\frac{m-2 \alpha}{\alpha}}\left((m-\alpha) \theta\left(t^{\frac{2}{\alpha}}\right)+2 t^{\frac{2}{\alpha}} \theta^{\prime}\left(t^{\frac{2}{\alpha}}\right)\right) .
\end{aligned}
$$

Thus $h^{\prime \prime}(t) \leq 0$ when $t \geq 0$ and condititon (26) holds. This yields the concavity of $h$ on the interval, and this concludes the proof.

We briefly illustrate Lemma 3.1 and Definition 3.1, by providing two examples of classic radial laws that are $\alpha$-revealed-concave for all $\alpha \in(-\infty, 1]$. 
Example 3.1 (Gaussian and Student random vectors) Let $\alpha$ be arbitrary and $\xi$ be a multivariate Gaussian random vector. Then elementary calculus shows that the generator (8) satisfies condition (25) (or (26) if $\alpha=0$ ). In particular, the associated radial distribution $F_{\mathcal{R}}$, (i.e., chi distribution with $m$ degrees of freedom) is $\alpha$-revealed-concave with

$$
\begin{array}{cc}
t(\alpha)=(m-\alpha)^{\frac{\alpha}{2}} & \text { if } \alpha \neq 0 \\
t(\alpha)=\log (m) / 2 & \text { if } \alpha=0 .
\end{array}
$$

Similarly, when $\xi \in \mathbb{R}^{m}$ is a multivariate Student random vector with $\nu$ degrees of freedom, then for $\alpha \in(-\nu, 1]$, its associated generator (9) satisfies condition (25) or (26). In particular, the associated radial distribution $F_{\mathcal{R}}$ is $\alpha$-revealed-concave with

$$
\begin{aligned}
t(\alpha)=\left(\frac{m-\alpha}{\alpha / \nu+1}\right)^{\alpha / 2} & \text { if } \alpha \neq 0 \\
t(\alpha)=\log (m) / 2 & \text { if } \alpha=0 .
\end{aligned}
$$

It is thus quite direct to show that the notion of "weak concavity" introduced at Definition 3.1 holds for these two distributions.

Many other promenant distribution functions can be shown to be $\alpha$-revealed-concave (for all $\alpha<0$ ). Indeed, [20] introduces the closely-related notion of $r$-decreasing density. In our words, [20, Lemma 3.1] shows that any distribution function disposing of an $(r+1)$-decreasing density is $\alpha$-revealed-concave for $\alpha=-r$. Thus, in particular, [20, Table 1] gathers many well-known distribution functions that are

\begin{tabular}{|c|c|c|c|}
\hline (Radial) Distribution & Parameters & $t(\alpha), \alpha \neq 0$ & $t(0)$ \\
\hline Chi & $m$ & $(\sqrt{m-\alpha})^{\alpha}$ & $\log (\sqrt{m})$ \\
\hline Chi-squared & $m$ & $(m-2 \alpha)^{\alpha}$ & $\log (m)$ \\
\hline Fisher-Snedecor & $m, \nu$ & $\left(\frac{m-\alpha}{\underline{\alpha}+1}\right)^{\alpha}($ provided $\alpha>-\nu)$ & $\log (\sqrt{m})$ \\
\hline Exponential & $\lambda$ & $\left(\frac{1-\alpha}{\lambda}\right)^{\alpha}$ & $\log \left(\frac{1}{\lambda}\right)$ \\
\hline Weibull & $a, b$ & $\left(\frac{b-\alpha}{a b}\right)^{\frac{\alpha}{b}}$ & $\log \left(\left(\frac{1}{a}\right)^{\frac{1}{b}}\right)$ \\
\hline Gamma & $a, b$ & $\left(\frac{a-\alpha}{b}\right)^{\alpha}$ & $\log \left(\frac{a}{b}\right)$ \\
\hline Log-Normal & $\mu, \sigma$ & $\left(\exp \left(\mu-\alpha \sigma^{2}\right)^{\alpha}\right)$ & $\mu$ \\
\hline Maxwell & $\sigma$ & $(\sigma \sqrt{3-\alpha})^{\alpha}$ & $\log (\sigma \sqrt{3})$ \\
\hline Rayleigh & $\lambda$ & $\left(\sqrt{\left(1-\frac{1}{2} \alpha\right) \lambda}\right)^{\alpha}$ & $\log (\sqrt{\lambda})$ \\
\hline
\end{tabular}
$\alpha$-revealed-concave. The situation of $\alpha \geq 0$ was not explicitly studied by the authors of [20], but in the vein of the above examples, [20, Table 1] can be completed to cover these situations too. Let us gather these relevant examples in Table 1.

Table 1 A collection of several $\alpha$-revealed concave mappings and associated parameter $t(\alpha)$.

\subsection{Generalized concavity of the ray function}

In this section, we show how convexity of $g$ in the first argument entails generalized concavity for the ray function $\rho$. We use here the standard notion of generalized concavity: we consider functions that show concavity or convexity when composed with a power or a logarithm function.

Definition 3.2 ( $\alpha$-concavity) For a given $\alpha \in[-\infty, 1]$, a non-negative mapping $f: \mathbb{R}^{n} \rightarrow \mathbb{R}_{+}$is called $\alpha$-concave whenever either one of the following conditions holds:

$-\alpha<0$ and $x \mapsto f^{\alpha}(x)$ is convex 
- $\alpha=0$ and $x \mapsto \log (f(x))$ is concave

$-\alpha>0$ and $x \mapsto f^{\alpha}(x)$ is concave.

Hence, 1-concavity amounts to classical concavity, 0-concavity is log-concavity (i.e., concavity of $\log f$ ), and $-\infty$-concavity identifies quasi-concavity. Generalized concavity can also be introduced more directly with the help of an auxiliary function (see [8, Definition 4.7]). Note that we restrict to $\alpha \leq 1$, because for $\alpha>1$ the notion is stronger than concavity and is not useful in our context. Note finally that the level sets of $\alpha$-concave fonctions are always convex.

The next lemma establishes that the $\alpha$-concavity of $\rho$ with respect to $x$ is automatic with $\alpha=-\infty$.

Lemma 3.2 (Quasi-concavity of the ray function) With the notation and under the assumptions of Theorem 2.1, for any $v \in \mathbb{S}^{m-1}$, the mapping $\rho(\cdot, v)$ is $-\infty$-concave. As a consequence, the uniform level set

$$
\operatorname{lev}_{\gamma}=\left\{x \in \mathbb{R}^{n}: g(x, \mu) \leq 0 \text { and } \rho(x, v) \geq \gamma \text { for all } v \in \mathbb{S}^{m-1}\right\}
$$

is convex (for any $\gamma)$.

Proof Pick $v \in \mathbb{S}^{m-1}, x_{1}, x_{2} \in D, \lambda \in[0,1]$ and define $x^{\lambda}:=\lambda x_{1}+(1-\lambda) x_{2}$. For $t \geq 0$, convexity $g$ in $x$ gives

$$
g\left(x^{\lambda}, \mu+t L v\right) \leq \lambda g\left(x_{1}, \mu+t L v\right)+(1-\lambda) g\left(x_{2}, \mu+t L v\right) .
$$

We will establish quasi-concavity of $\rho(\cdot, v)$, i.e.,

$$
\rho\left(x^{\lambda}, v\right) \geq \min \left(\rho\left(x_{1}, v\right), \rho\left(x_{2}, v\right)\right)
$$

using its expression (19) as a sup over $t$. We distinguish three cases:

Case 1: $\rho\left(x_{1}, v\right)<\infty$ and $\rho\left(x_{2}, v\right)<\infty$. From (19), we have $g\left(x_{1}, \mu+t L v\right) \leq 0$ for any $t \leq \rho\left(x_{1}, v\right)$ (and likewise for $\left.x_{2}\right)$. Thus for any $t \leq \min \left(\rho\left(x_{1}, v\right), \rho\left(x_{2}, v\right)\right)$ we have $g\left(x^{\lambda}, \mu+t L v\right) \leq 0$ by (28). Consequently (29) holds.

Case 2: either $\rho\left(x_{1}, v\right)=\infty$ or $\rho\left(x_{2}, v\right)=\infty$. Switching the role of $x_{1}$ and $x_{2}$, we can assume that $\rho\left(x_{1}, v\right)=\infty$ which gives that $g\left(x_{1}, \mu+t L v\right) \leq 0$ for all $t \geq 0$. For any $t \leq \rho\left(x_{2}, v\right)$ such that $g\left(x_{2}, \mu+t L v\right) \leq 0$, we have $g\left(x^{\lambda}, \mu+t L v\right) \leq 0$ by (28). This gives $\rho\left(x^{\lambda}, v\right) \geq \rho\left(x_{2}, v\right)$ and therefore (29) holds too.

Case 3: $\rho\left(x_{1}, v\right)=\infty$ and $\rho\left(x_{2}, v\right)=\infty$. For any $t \geq 0$, we have $g\left(x_{1}, \mu+t L v\right) \leq 0, g\left(x_{2}, \mu+t L v\right) \leq 0$ and then $g\left(x^{\lambda}, \mu+t L v\right) \leq 0$ by (28). Hence, $\rho\left(x^{\lambda}, v\right)=\infty$ and (29) holds too.

In the three cases, (29) holds, which establishes the quasi-concavity. The convexity of $\operatorname{lev}_{\gamma}$ then follows as it is the (possibly empty) intersection of level-sets of the convex function $g(\cdot, \mu)$ and of the quasi-concave functions $\rho(\cdot, v)$.

Although, this result states that $-\infty$-concavity is automatic, we will need a slightly stronger assumption, namely $\alpha$-concavity with $\alpha>-\infty$. This slightly stronger assumption, will help guarantee concavity of the probability function on an appropriate set. Despite the fact that this stronger assumption is not automatic in general, we will see in Section 5 that this property holds for many useful functions $g$.

\subsection{Restricted concavity of the probability function}

The generalized concavity of $\rho$ and the concavity-revealing $F_{\mathcal{R}}$, established in the previous two sections, allow us to prove some concavity of $\varphi$ on a special convex set. 
Theorem 3.1 (Restricted concavity of $\varphi$ ) Under the assumptions of Theorem 2.1, assume that there exists $\alpha>-\infty$ such that $\rho(\cdot, v)$ is $\alpha$-concave for all $v \in \mathbb{S}^{m-1}$ (see Definition 3.2). Assume furthermore that $F_{\mathcal{R}}$ is $\alpha$-revealed-concave with associated $t(\alpha)$ (see Definition 3.1), and introduce $t^{*}(\alpha)>0$ by

$$
t^{*}(\alpha):=t(\alpha)^{\frac{1}{\alpha}} \quad \text { for } \alpha \neq 0 \quad \text { and } \quad t^{*}(0):=\exp (t(0)) .
$$

Then the probability function $\varphi$ is concave on $E(\alpha):=\operatorname{lev}_{t^{*}(\alpha)}$, the uniform level set, defined in $(27)$, associated to $t^{*}(\alpha)$.

Proof We notice that $E(\alpha)$ is convex by Lemma 3.2. We then prove the concavity of $\varphi$ on $E(\alpha)$ for $\alpha<0$; the proofs for two other cases $\alpha=0$ and $\alpha>0$ follow the same lines. Fix $v \in \mathbb{S}^{m-1}$, pick $x_{1}, x_{2} \in E(\alpha)$ and $\lambda \in[0,1]$. We note first that

$$
\lambda \rho\left(x_{1}, v\right)^{\alpha}+(1-\lambda) \rho\left(x_{2}, v\right)^{\alpha} \leq \max \left\{\rho\left(x_{1}, v\right)^{\alpha}, \rho\left(x_{2}, v\right)^{\alpha}\right\} \leq t(\alpha) .
$$

To establish the second inequality above, note that $x_{1} \in E(\alpha)$, i.e., $\rho\left(x_{1}, v\right) \geq t^{*}(\alpha)$ and hence from $\alpha<0$ we deduce $\rho\left(x_{1}, v\right)^{\alpha} \leq t^{*}(\alpha)^{\alpha}=t(\alpha)$. We can do the same for $x_{2}$ and then (30) follows. We write now, for $x^{\lambda}=\lambda x_{1}+(1-\lambda) x_{2}$,

$$
F_{\mathcal{R}}\left(\rho\left(x^{\lambda}, v\right)\right) \geq F_{\mathcal{R}}\left(\left(\lambda \rho\left(x_{1}, v\right)^{\alpha}+(1-\lambda) \rho\left(x_{2}, v\right)^{\alpha}\right)^{\frac{1}{\alpha}}\right),
$$

by monotonicity of $F_{\mathcal{R}}$ and $\alpha$-concavity of $\rho(\cdot, v)$. Since $F_{\mathcal{R}}$ is $\alpha$-revealed-concave, we get

$$
F_{\mathcal{R}}\left(\rho\left(x^{\lambda}, v\right)\right) \geq \lambda F_{\mathcal{R}}\left(\rho\left(x_{1}, v\right)\right)+(1-\lambda) F_{\mathcal{R}}\left(\rho\left(x_{2}, v\right)\right)
$$

with the help of (30). Since this holds for all $v \in \mathbb{S}^{m-1}$, we integrate over $\mathbb{S}^{m-1}$ and obtain from (18) that:

$$
\begin{aligned}
\varphi\left(x^{\lambda}\right) & =\int_{v \in \mathbb{S}^{m-1}} F_{\mathcal{R}}\left(\rho\left(x^{\lambda}, v\right)\right) d \mu_{\zeta}(v) \\
& \geq \lambda \int_{v \in \mathbb{S}^{m-1}} F_{\mathcal{R}}\left(\rho\left(x_{1}, v\right)\right) d \mu_{\zeta}(v)+(1-\lambda) \int_{v \in \mathbb{S}^{m-1}} F_{\mathcal{R}}\left(\rho\left(x_{2}, v\right)\right) d \mu_{\zeta}(v) \\
& =\lambda \varphi\left(x_{1}\right)+(1-\lambda) \varphi\left(x_{2}\right)
\end{aligned}
$$

which proves the concavity of $\varphi$ on $E(\alpha)$.

We end this section with brief illustrations of the previous result.

Example 3.2 (Gaussian and Student again) Let $\xi \in \mathbb{R}^{m}$ be a Gaussian random vector and assume that there exists an $\alpha \in(-\infty, 1]$ such that for any $v \in \mathbb{S}^{m-1}, \rho(\cdot, v)$ is $\alpha$-concave. We can then apply Theorem 3.1 to the associated distribution function $F_{\mathcal{R}}$. In view of Example 3.1, we can also establish that $t^{*}(\alpha)=\sqrt{m-\alpha}$ (for both $\alpha=0$ or $\neq 0$ ). Thus we obtain that the mapping $\varphi$ is concave on the convex set $E(\alpha)=\operatorname{lev}_{\sqrt{m-\alpha}}$. Similarly, if $\xi \in \mathbb{R}^{m}$ is a multivariate Student random and if that there exists an $\alpha \in(-\nu, 1]$ such that for any $v \in \mathbb{S}^{m-1}, \rho(\cdot, v)$ is $\alpha$-concave, then the mapping $\varphi$ is concave on the convex set $E(\alpha)=\operatorname{lev} \sqrt{\frac{m-\alpha}{\frac{\alpha}{\nu}+1}}$.

Example 3.3 (Gaussian multivariate distribution) Let us consider the special function $g(x, z)=z-x$ which correspond to the situation of a multivariate distribution function. Through elementary computation, we have

$$
\rho(x, v)=\min _{i \in\left\{j=1, \ldots, m:(L v)_{j}>0\right\}} \frac{x_{i}-\mu_{i}}{(L v)_{i}} \quad \text { if } L v \geq 0 \quad \text { and }+\infty \text { otherwise. }
$$

which is clearly concave. Theorem 3.1 then gives that the multivariate Gaussian distribution function is concave on the set $\left\{x \in \mathbb{R}^{m}: x \geq \mu+\sqrt{m-1}\|L\| e\right\} \subseteq \operatorname{lev}_{\sqrt{m-1}}$ (with $e$ the all-one vector). Thus [41, Theorem 2.1] comes as a special case of Theorem 3.1 above. Note finally that it is well known that the multivariate Gaussian distribution function is log-concave. 


\section{Eventual convexity of probability constraints}

In this section, we state the main result of the paper, which provides an expression of the threshold on $p$ from which the convexity of the probability constrained set $M(p)(6)$ is guaranteed in a general situation. We then refine our analysis when considering special cases as corollaries.

Theorem 3.1, in the previous section, establishes the concavity of $\varphi$ when restricted to $E(\alpha)$. We show now that $M(p) \subseteq E(\alpha)$ for $p$ large enough, under an additional assumption on $g$, and this gives us the convexity of the probability-constrained set $M(p)$. This assumption involves $\rho^{\text {co }}: \mathbb{R}^{n} \times \mathbb{S}^{m-1} \rightarrow[-\infty,+\infty]$ the ray function associated with the convex hull of $\mathfrak{M}(x)(4)$, defined, similarly to $\rho$ in (19), by

$$
\rho^{\mathrm{co}}(x, v):=\rho_{\operatorname{co} \mathfrak{M}(x)}(v)=\left\{\begin{array}{l}
\sup _{t \geq 0} \quad t \\
\text { s.t. }
\end{array} \quad \mu+t L v \in \operatorname{Co} \mathfrak{M}(x) .\right.
$$

Note that, by definition of $\rho^{\mathrm{co}}$ and $\rho$, we have

$$
\rho^{\mathrm{co}}(x, v) \geq \rho(x, v) \quad \text { for all } x \in \mathbb{R}^{n} \text { and } v \in \mathbb{S}^{m-1} .
$$

The addition assumption is that we also have the converse inequality up to factor: see condition (32) in the next theorem.

Theorem 4.1 (General eventual convexity) Let $\xi$ be an elliptical random vector and $g: \mathbb{R}^{n} \times \mathbb{R}^{m} \rightarrow \mathbb{R}^{k}$ be a lower semi-continuous mapping that is convex with respect to the first argument. Assume that there exists $\alpha \in \mathbb{R}$ such that $F_{\mathcal{R}}$ is $\alpha$-revealed-concave (Definition 3.1) and that for each $v \in \mathbb{S}^{m-1}, \rho(\cdot, v)$ is $\alpha$-concave (Definition 3.2). Assume furthermore that for a given $p_{0} \in\left(\frac{1}{2}, 1\right)$, there exists a $\delta^{\text {nd }}>0$ such that

$$
\delta^{n d} \rho(x, v) \geq \rho^{\mathrm{co}}(x, v) \quad \text { for all } x \in M\left(p_{0}\right) \text { and } v \in \mathbb{S}^{m-1} .
$$

Then the probability constrained set

$$
M(p)=\left\{x \in \mathbb{R}^{n}: \quad \mathbb{P}[g(x, \xi) \leq 0] \geq p\right\}
$$

is convex when $p$ is large enough; more precisely, for any given $q \in\left(0, \frac{1}{2}\right), M(p)$ is convex when $p \geq$ $\max \left\{p(\alpha, q), p_{0}\right\}$ with

$$
p(\alpha, q):=\left(\frac{1}{2}-q\right) F_{\mathcal{R}}\left(\frac{t^{*}(\alpha) \delta^{\text {nd }}}{\delta(q)}\right)+\frac{1}{2}+q,
$$

where $\delta(q)>0$ is given by Lemma 2.3 and $t^{*}(\alpha)>0$ as in Theorem 3.1.

Proof Consider $p \in\left(p_{0}, 1\right)$, and take $x \in M(p) \subseteq M\left(p_{0}\right)$ arbitrarily. We have

$$
\frac{1}{2}<p \leq \mathbb{P}[g(x, \xi) \leq 0] \leq \mathbb{P}[\xi \in \operatorname{Co} \mathfrak{M}(x)] .
$$

Corollary 2.1 then gives that $\mu \in \operatorname{int} \operatorname{Co} \mathfrak{M}(x)$. Let us now pick an arbitrary but fixed $v \in \operatorname{dom}(\rho(x, \cdot))$. Note that, due to (32), it follows that $v \in \operatorname{dom}\left(\rho^{\mathrm{co}}(x, \cdot)\right)$ as well. Thus we have

$$
\mu+\rho^{c o}(x, v) L v \in \operatorname{bd} \operatorname{Co}(\mathfrak{M}(x)),
$$

by definition $(31)$ of $\rho^{c o}$. We can separate $\mu+\rho^{c o}(x, v) L v$ from the convex set $\operatorname{Co}(\mathfrak{M}(x))$ : there exists a nonzero $s$ such that for all $z \in \operatorname{Co}(\mathfrak{M}(x))$

$$
s^{\top} z \leq s^{\top}\left(\mu+\rho^{\mathrm{co}}(x, v) L v\right) \leq s^{\top}\left(\mu+\delta^{\text {nd }} \rho(x, v) L v\right),
$$

the second inequality above coming from the fact that $g$ satisfies (32) (together with $s^{\top} L v \geq 0$ since $\mu$ lies in $\operatorname{Co}(\mathfrak{M}(x)))$. Define

$$
c^{\top} z \leq \gamma \quad \text { with } \quad c=\frac{s}{\left\|L^{\top} s\right\|} \quad \text { and } \quad \gamma=c^{\top}\left(\mu+\delta^{\text {nd }} \rho(x, v) L v\right),
$$


where we recall that $\left\|L^{\top} s\right\|>0$, since $L$ is regular and $s \neq 0$. It now follows that

$$
\mathfrak{M}(x) \subseteq \operatorname{Co}(\mathfrak{M}(x)) \subseteq\left\{z \in \mathbb{R}^{m}: c^{\top} z \leq \gamma\right\} .
$$

In particular this entails $\mathbb{P}[g(x, \xi) \leq 0] \leq \mathbb{P}\left[c^{\top} \xi \leq \gamma\right]$. We can employ Theorem 2.2 to get for any $q \in\left(0, \frac{1}{2}\right)$ and associated $\delta(q)>0$ that

$$
\begin{aligned}
p \leq \mathbb{P}[g(x, \xi) \leq 0] & \leq \mathbb{P}\left[c^{\top} \xi \leq \gamma\right] \\
& \leq\left(\frac{1}{2}-q\right) F_{\mathcal{R}}\left(\frac{\delta^{\mathrm{nd}} \rho(x, v) \frac{s^{\top} L v}{\left\|L^{\top} s\right\|}}{\delta(q)}\right)+q+\frac{1}{2} \\
& \leq\left(\frac{1}{2}-q\right) F_{\mathcal{R}}\left(\frac{\delta^{\mathrm{nd}} \rho(x, v)}{\delta(q)}\right)+q+\frac{1}{2},
\end{aligned}
$$

where we used the Cauchy-Schwarz inequality, the fact that $v \in \mathbb{S}^{m-1}$, and monotonicity of $F_{\mathcal{R}}$. Inverting this inequality, we obtain

$$
\rho(x, v) \geq \frac{\delta(q)}{\delta^{\text {nd }}} F_{\mathcal{R}}^{-1}\left(\frac{p-q-\frac{1}{2}}{\frac{1}{2}-q}\right) .
$$

The above inequality has been established for an arbitrary but fixed $v \in \operatorname{dom}(\rho(x, \cdot))$, and it obviously extends to $v \notin \operatorname{dom}(\rho(x, \cdot))$. Thus for any $p \geq p(\alpha, q)$, we have $\rho(x, v) \geq t^{*}(\alpha)$ hence $M(p) \subseteq E(\alpha)$. Theorem 3.1 allows us to conclude that $\varphi$ is concave on $E(\alpha)$ and therefore $M(p)$ is convex for $p \geq$ $\max \left\{p(\alpha, q), p_{0}\right\}$.

Theorem 4.1 establishes the convexity of the constrained set $M(p)$ under assumptions that correspond to

(i) generalized convexity properties of the law of $\xi$ : namely, that the radial distribution function $F_{\mathcal{R}}$ is $\alpha$-revealed-concave;

(ii) geometrical properties of $g$ : namely, that $\rho$ is uniformly $\alpha$-concave and that condition (32) holds.

These assumptions, though appearing abstract, are not too restrictive in practice. The condition on $F_{\mathcal{R}}$ is easy to obtain with the help of Lemma 3.1. The $\alpha$-concavity of $\rho$ can often be obtained from the explicit expressions of $\rho$ as in the examples of Section 5 .

The last condition (32) although formulated in an abstract way with an can be readily verified from $g$ in various concrete situations: when $\mathfrak{M}(x)$ is bounded, or when $g$ is convex in the second argument (though not necessary jointly-convex, so that the case (3) is covered). We provide below some corollaries corresponding to these cases.

Corollary 4.1 (When $g$ is convex with respect to each variable) Let $\xi$ be an elliptical random vector and $g: \mathbb{R}^{n} \times \mathbb{R}^{m} \rightarrow \mathbb{R}^{k}$ be a lower semi-continuous mapping that is convex with respect to the first and second argument argument (but possibly not jointly). Assume that there exists $\alpha \in \mathbb{R}$ such that $F_{\mathcal{R}}$ is $\alpha$-revealed-concave and that for each $v \in \mathbb{S}^{m-1}, \rho(\cdot, v)$ is $\alpha$-concave. Then, for any given $q \in\left(0, \frac{1}{2}\right)$, the probability constrained set $M(p)$ is convex provided that

$$
p \geq\left(\frac{1}{2}-q\right) F_{\mathcal{R}}\left(\frac{t^{*}(\alpha)}{\delta(q)}\right)+\frac{1}{2}+q,
$$

Proof If $g$ is convex in the second argument, then $\mathfrak{M}(x)$ is convex and therefore $\rho^{c o}$ and $\rho$ coincide. Thus (32) holds for $p_{0}=\frac{1}{2}$ and $\delta^{\text {nd }}=1$. Note that we also have (17) directly. The corollary is thus just the specification of Theorem 4.1 to this case; the expression of the threshold is (33) with $\delta^{\text {nd }}=1$. 
Corollary 4.2 (Under boundedness assumption) Let $\xi$ be an elliptical random vector and $g: \mathbb{R}^{n} \times$ $\mathbb{R}^{m} \rightarrow \mathbb{R}^{k}$ be a lower semi-continuous mapping that is convex with respect to the first argument. Assume given a compact set $X$ such that, for all $x \in X, g(x, \mu)<0$ and $\left\{z \in \mathbb{R}^{m}: g(x, z) \leq 0\right\}$ is bounded. Let furthermore $\alpha \in \mathbb{R}$ be such that $F_{\mathcal{R}}$ is $\alpha$-revealed-concave and that for each $v \in \mathbb{S}^{m-1}, \rho(., v)$ is $\alpha$-concave. Then the probability constrained set $M(p) \cap X$ is convex for all $p \geq p(\alpha, q)$ given by (33).

Proof Let us fix $\bar{x} \in X$. Since we have $g_{j}(\bar{x}, \mu)<0$ for all $j=1, \ldots, k$, the continuity of $g$ provides a $r>0$ and a neighbourhood $U$ of $\bar{x}$ such that

$$
g_{j}\left(x, \mu+r^{\prime} L v\right)<0 \quad \text { for all } r^{\prime}<r, x \in U .
$$

This means that $B(\mu, r) \subseteq \mathfrak{M}(x)$ for all $x \in U$. On the other hand, by arguments equivalent to those of [54, Proposition 3.13] it follows that $\mathfrak{M}(x)$ is bounded for all $x \in U$ (shrinking $U$ if necessary). According to [2, Theorem 3.1.3] we can find some $R$ such that $\mathfrak{M}(x) \subseteq$ Co $\mathfrak{M}(x) \subseteq B(\mu, R)$ for all $x \in U$. Thus there exists constants $R>r>0$ such that $B(\mu, r) \subseteq \mathfrak{M}(x) \subseteq \operatorname{Co} \mathfrak{M}(x) \subseteq B(\mu, R)$ for all $x$. This yields that (32) holds for all $x \in U$ for $p_{0}=\frac{1}{2}$ and $\delta^{\text {nd }} \leq \frac{R}{r}$. Indeed, for any $x \in \mathbb{R}^{m}$ and $v \in \mathbb{S}^{m-1}$, we have $r \leq \rho(x, v)$ since $B(\mu, r) \subseteq \mathfrak{M}(x)$, but also $\mathfrak{M}(x) \subseteq \operatorname{Co} \mathfrak{M}(x) \subseteq B(\mu, R)$. Consequently $\rho^{\mathrm{co}}(x, v) \leq R$, and then (32) holds with a $\delta^{\text {nd }} \leq \frac{R}{r}$.

We can now conclude by repeating this argument for each $\bar{x} \in X$ to get a neighbourhood $U$ of $\bar{x}$ and a $\delta_{\bar{x}}^{\text {nd }}>0$ such that (32) holds true. By a compactness argument, we can thus assume that (32) holds for all $x \in M\left(\frac{1}{2}\right) \cap X$. The statement now follows from Theorem 4.1.

Let us mention an example of (indirect) application of this corollary in optimization.

Example 4.1 (Random vector with bounded support) Consider the following optimization problem

$$
\begin{array}{rl}
\min _{x \in X} & f(x) \\
\text { s.t. } & \mathbb{P}[g(x, \xi) \leq 0] \geq p,
\end{array}
$$

where $f$ is a convex function and $X$ is a compact convex set. We assume that the random variable $\xi$ is an elliptically distributed random vector with compact support; which means that the generator $\theta$ of (7) is such that there exists a $\bar{t}>0$ for which $\theta(t)=0$ for all $t \geq \bar{t}$. In this situation, Corollary 4.2 can guarantee that we have a convex optimization for $p$ large enough. The reasoning is as follows.

Introduce the restricting map $\hat{g}$ defined in the spherical-radial variable by

$$
\hat{g}(x, \mu+t L v)=\left\{\begin{array}{ccc}
g(x, \mu+t L v) & \text { if } & t \leq \bar{t} \\
g(x, \mu+t L v) & \text { if }
\end{array} \quad g(x, \mu+t L v)>0 \text { and } t \geq \bar{t}\right.
$$

The compact support of $\xi$ implies by (11) that $F_{\mathcal{R}}(r)=0$ for $r \geq \sqrt{\bar{t}}$. This yields that we have by (18) the identification

$$
\varphi(x):=\mathbb{P}[g(x, \xi) \leq 0]=\mathbb{P}[\hat{g}(x, \xi) \leq 0] .
$$

The interest of considering $\hat{g}$ appears now: the set

$$
\left\{z \in \mathbb{R}^{m}: \hat{g}(x, z) \leq 0\right\} \subseteq\left\{z \in \mathbb{R}^{m}:\|z\| \leq \bar{t}\|L v\| \text { for some } v \in \mathbb{S}^{m-1}\right\}
$$

is bounded, which is the main assumption of Corollary 4.2. Observe also the other assumptions hold in our setting. Indeed, the lower semi-continuity of $g$ is transferred to $\hat{g}$, the star-shapedness of $\mathfrak{M}(x)$ is transferred to $\left\{z \in \mathbb{R}^{m}: \hat{g}(x, z) \leq 0\right\} \subseteq \mathfrak{M}(x)$, and the $\alpha$-concavity is transferred automatically. Thus Corollary 4.2 to assert convexity of $M(p) \cap X$ for $p$ large enough.

We finish this section by yet another corollary of Theorem 4.1 to emphase that the estimates used to get the general expression of $p(\alpha, q)$ can be refined in some special cases. 
Corollary 4.3 (In the Gaussian case) In the setting of Theorem 4.1, when $\xi \sim \mathcal{N}(\mu, \Sigma)$, the condition on the radial distribution function $F_{\mathcal{R}}$ is automatically verified. Under the remaining conditions, the set $M(p)$ is convex if $p \geq \max \left\{\Phi\left(\delta^{n d} \sqrt{m-\alpha}\right), p_{0}\right\}$, where $\Phi$ is the standard one-dimension Gaussian distribution function.

Proof Recall from from Example 3.2, that the associated radial distribution of $\xi$ is a chi distribution with $m$ degrees of freedom, which is $\alpha$-revealed-concave for all $\alpha$ with associated parameter $t^{*}(\alpha)=\sqrt{m-\alpha}$. Moreover, note that $c^{\top} \xi \sim \mathcal{N}\left(c^{\top} \mu, c^{\top} \Sigma c\right)$, hence equation (34) can be replaced by:

$$
\mathbb{P}\left[c^{\top} \xi \leq \gamma\right]=\Phi\left(\frac{\gamma-c^{\top} \mu}{c^{\top} \Sigma c}\right) \leq \Phi\left(\frac{\delta^{\text {nd }} \rho(x, v)\left|c^{\top} L v\right|}{\sqrt{c^{\top} \Sigma c}}\right) .
$$

Consequently:

$$
\rho(x, v) \geq \frac{\Phi^{-1}(p) \sqrt{c^{\top} \Sigma c}}{\delta^{\text {nd }}\left|c^{\top} L v\right|} .
$$

The Cauchy-Schwartz inequality yields: $\left|c^{\top} L v\right| \leq\left\|c^{\top} L\right\|\|v\|$ and we also have $\sqrt{c^{\top} \Sigma c}=\left\|c^{\top} L\right\|=1$. By combining these inequalities with (36) we get:

$$
\rho(x, v) \geq \Phi^{-1}(p) \frac{1}{\delta^{\text {nd }}}>0,
$$

which leads to $\rho(x, v) \geq t^{*}(\alpha)$ by our definition of $p$. The rest of the arguments of Theorem 4.1 can be carried out verbatim.

\section{Illustrative examples}

The previous section provides convexity results for probabilistically constrained sets in a quite general setting. In this section, we specify these results on relevant cases in the literature, by giving explicit convexity statements with computable convexity certificates, readily evaluated from the nominal problem data. The first situation is related to the study of linear probability constraints with random technology matrices; the second for some quadratic constraints arising in power systems.

\subsection{Elliptically distributed technology matrices}

We look closely here to the case of affine probability constraints with random technology matrices, encompassing the motivating example (3). The situation essentially refers to the case wherein the system described by $g$ is polyhedral in $z$. The terminology dates back at least to [39] and is also used in $[25,26]$ so that we adopt it too. We recall that convexity results for this case exist whenever either the matrix has independent rows (see e.g., [20,51]) or the covariance matrix has a special structure (see [42, Theorem 2.19, Theorem 2.20]) but only when $\xi$ follows a multivariate Gaussian distribution function. The tools provided in this paper allow us to extend the set of cases captured, in two directions: by allowing for arbitrary covariance matrices and for elliptically distributed matrices. To illustrate the type of results we can obtain, we first write a formal proposition for the case of Gaussian vectors and then give a specific example for Student vectors.

Proposition 5.1 Consider the probability function $\varphi(x)=\mathbb{P}[\Xi x \leq \beta]$, where $\beta \in\left(\mathbb{R}_{+}^{*}\right)^{m}$ and $\Xi$ is a $m \times n$ centered (i.e., of zero mean) multi-variate Gaussian random matrix. Then the probability constrained set $M(p)=\left\{x \in \mathbb{R}^{n}: \varphi(x) \geq p\right\}$ is convex for all $p \geq \Phi(\sqrt{m n+1})$, where $\Phi$ is the distribution function of a one-dimensional standard normal Gaussian random variable. 
Proof It is convenient to reformulate $\varphi$ by vectorizing $\Xi$. To this end, we identify $\Xi \in \mathbb{R}^{m \times n}$ with the vector $\xi \in \mathbb{R}^{m n}$ obtained by concatenating its columns, and we introduce the mapping $T: \mathbb{R}^{n} \rightarrow \mathbb{R}^{m \times(m n)}$ :

$$
T(x)=\left(\begin{array}{cccc}
x^{\top} & 0 & \cdots & 0 \\
0 & x^{\top} & \cdots & 0 \\
\vdots & \cdots & \cdots & \vdots \\
0 & 0 & \cdots & x^{\top}
\end{array}\right)
$$

It then readily follows that $\varphi(x)=\mathbb{P}[T(x) \xi \leq \beta]$. Note also that the covariance matrix $\Sigma$ of $\xi$ is a positive definite of size $m n \times m n$. Thus $\eta(x):=\Xi x=T(x) \xi$ follows a multi-variate Gaussian distribution with mean $\mu(x)=T(x) 0 \in \mathbb{R}^{m}$ and $m \times m$ covariance matrix $\Sigma(x)=T(x) \Sigma T(x)^{\top}$. The entries of $\Sigma_{i j}(x)$ can be written for any $1 \leq i, j \leq m$ as $\Sigma_{i j}(x)=x^{\top} \Sigma^{i j} x$, where $\Sigma^{i j}$ is the $n \times n$ matrix defined as $\Sigma_{l k}^{i j}=\Sigma_{(i-1) n+l,(j-1) n+k}, 1 \leq l, k \leq n$.

We define the mapping $g: \mathbb{R}^{n} \times \mathbb{R}^{m n} \rightarrow \mathbb{R}^{m}$ as $g(x, z):=T(x) z-\beta$. It follows that $\varphi(x)=\mathbb{P}[g(x, \xi) \leq 0]$ and that $g$ is convex in $x$ and in $z$ separately. We now turn our attention to the $\alpha$-concavity of the mapping $\rho$ of (19). Looking at the system $g(x, \mu+r L v) \leq 0$ component-wise, we get

$$
\rho(x, v)=\min \left\{\rho_{1}(x, v), \ldots, \rho_{m}(x, v)\right\}
$$

where the $\rho_{i}$ are defined with the help of $T_{i}(x)$ the $i$ th row of matrix $T(x)$ by

$$
\rho_{i}(x, v):=\left\{\begin{array}{cl}
\frac{\beta_{i}}{T_{i}(x) L v} & \text { when } T_{i}(x) L v>0 \\
-\infty & \text { else }
\end{array}\right.
$$

Since the map $\rho_{i}(\cdot, v)$ is -1-concave, we get from $[8$, Theorem 4.21]) that $\rho(\cdot, v)$ is -1-concave as well. Thus Corollary 4.1 (and Corollary 4.3 in the Gaussian case) gives the results, since the mapping $g$ is convex in the second argument.

Similar results can be established for other elliptical distribution. For example, with a multi-variate Student distribution instead of Gaussian in the above proposition, we would get that for any $q \in\left(0, \frac{1}{2}\right)$, the set $M(p)=\left\{x \in \mathbb{R}^{n}: \varphi(x) \geq p\right\}$ is convex for all

$$
p \geq\left(\frac{1}{2}-q\right) F_{m n, \nu}\left(\frac{\nu(m n+1)}{\delta(q)^{2}(m n \nu-m n)}\right)+q+\frac{1}{2}
$$

where $F_{m n, \nu}$ is the Fisher-Snedecor distribution with $m n$ and $\nu$ degrees of freedom respectively. Let us illustrate the result with the concrete data in two dimension with $m=n=\nu=2$ and $\beta=1$ and the concrete covariance matrix

$$
\Sigma=\left[\begin{array}{cccc}
1.00 & -0.75 & -0.16 & 0.11 \\
-0.75 & 1.00 & 0.13 & 0.11 \\
-0.16 & 0.13 & 1.00 & 0.23 \\
0.11 & 0.11 & 0.23 & 1.00
\end{array}\right]
$$

For any $q \in\left(0, \frac{1}{2}\right)$, with the help of Lemma 2.3, the value $\delta(q)$ and therefore the threshold (39) can be computed. We can even minimize the right-hand side over $q$ to obtain the best threshold. By doing so numerically we obtain $q^{*}=0.2391$ and associated $p^{*}=0.8263$. Figure 1 shows the contour lines of the probability function and illustrates that $p^{*}$ is a conservative estimate in this case, since convexity of level sets starts failing around $p=1 / 2$. Still, $p^{*}$ is not large and clearly exploitable in practice where values $p \approx 0.9$ for probability constraints are commonplace. 


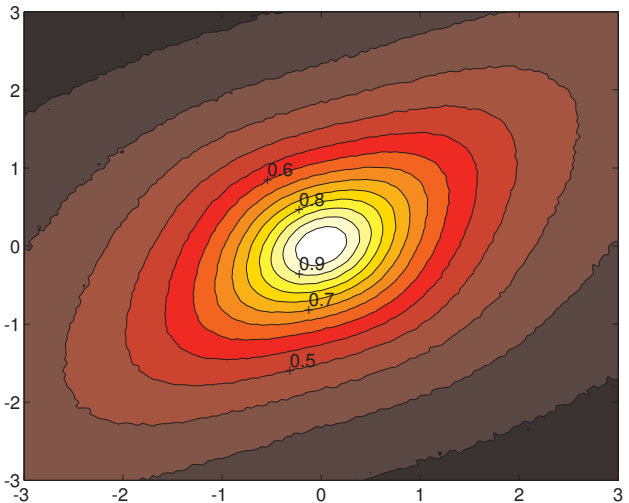

Fig. 1 Contour lines of a probability function involving a correlated Student distribution technology matrix

5.2 Quadratic probability constraints arising in power systems

We investigate here the situation studied by the recent paper [31], which is relevant for optimal power flow problems. We consider the probability constraint $\varphi(a, c) \geq p$ where $(a, c) \in \mathbb{R}^{m} \times \mathbb{R}^{m}$ are decision vectors and

$$
\varphi(a, c)=\mathbb{P}\left[\left(a^{\top} \xi\right)^{2}+\left(c^{\top} \xi\right)^{2} \leq k\right],
$$

with $\xi \sim \mathcal{N}(0, \Sigma)$ a multivariate Gaussian random vector. Such probability constraint appear when extending standard models of optimal power flow problems to account for uncertainty and reactive power; see [31, Chap. 2]. Eventual convexity of the feasible set of constraints of the type (40) is conjectured in [31] but the authors mention not to be able to establish it. With the tools developed in this paper, we can now obtain the following convexity certificate for $p$ large enough.

Proposition 5.2 Consider the mapping $\varphi$ of (40) with an elliptical random vector $\xi$ and assume that the associated radial distribution $F_{\mathcal{R}}$ is $\alpha$-revealed-concave for $\alpha=-2$. Then for any $q \in\left(0, \frac{1}{2}\right)$, the set $M(p)=\{(a, c): \varphi(a, c) \geq p\}$ is convex provided that

$$
p \geq\left(\frac{1}{2}-q\right) F_{\mathcal{R}}\left(\frac{t^{*}(-2)}{\delta(q)}\right)+q+\frac{1}{2} .
$$

If $\xi \sim \mathcal{N}(0, \Sigma)$, then this latter set is convex provided that $p \geq \Phi(\sqrt{m+2})$.

Proof Consider the mapping $g(a, c, z)=\left(a^{\top} z\right)^{2}+\left(c^{\top} z\right)^{2}-k$. The mapping $g$, as a composition of affine mappings with the convex function $\left(x_{1}, x_{2}\right) \mapsto x_{1}^{2}+x_{2}^{2}-k$ is convex in $(a, c)$ and too in $z$. By solving $g(a, c, r L v)=0$ in $r$, we get that the resolvent mapping $\rho(x, v)$ as defined in (19) is given by

$$
\rho(a, c, v)=\sqrt{\frac{k}{\left(a^{\top} L v\right)^{2}+\left(c^{\top} L v\right)^{2}}},
$$

For any fixed $v$, it is immediately observed that $\rho(a, c, v)^{-2}$ is convex i.e., $\rho(a, c, v)$ is -2 -concave. We can use Theorem 4.1 (or Corollary 4.1 for Gaussian $\xi$ ) to establish convexity of $M(p)$.

Let us finally illustrate the above proposition with a specific case taken from [31]: consider (40) with $m=2, a=(x, 0), c=(0, y), k=1$ and $\xi \sim \mathcal{N}(0, I)$. The bound of the above proposition is $p^{*}=0.97725$ in this case. We can thus guarantee the convexity of the constraint for $p \geq 0.97725$. Note that it is proved in [31] that the constraint is not convex for $p=0.545$ (by showing that the points $(0.6,1.0)$ and $(1.0,0.6)$ belong to $M(0.545)$, but $(0.8,0.8)$ does not $)$. 


\section{Conclusions}

In this paper we have provided conditions under which probability constraints define a convex set. These conditions can be readily verified from the nominal problem data and are more general than those appearing in similar prior results. Several corollaries and examples show the interest of our study.

In the illustrative numerical examples, we have observed a gap between the asserted convexity threshold and the empirical one. This is expected, since our results have not only shown convexity of upper levelsets for probability functions, but also that the probability function is concave on them, for a probability value large enough. Establishing the quasi-concavity of the probability function on a larger set is still an open question. In future work, we will investigate this as well as other ways to lower the certificate provided here.

Acknowledgements We are thankful for the constructive comments from two anonymous referees and the associate editor. We would like to acknowledge the financial support of PGMO (Gaspard Monge Program for Optimization and operations research) of the Hadamard Mathematic Foundation, through the project "Advanced nonsmooth optimization methods for stochastic programming".

\section{References}

1. Arnold, T., Henrion, R., Möller, A., Vigerske, S.: A mixed-integer stochastic nonlinear optimization problem with joint probabilistic constraints. Pacific Journal of Optimization 10, 5-20 (2014)

2. Bank, B., Guddat, J., Klatte, D., Kummer, B., Tammer, K.: Non-Linear Parametric Optimization. Birkhäuser Basel (1982)

3. Ben-Tal, A., Nemirovski, A.: Robust solutions of linear programming problems contaminated with uncertain data. Mathematical Programming, series A 88, 411-424 (2000)

4. Brascamp, H., Lieb, E.: On extensions of the Brunn-Minkowski and Prékopa-Leindler theorems, including inequalities for log-concave functions and with an application to the diffusion equations. Journal of Functional Analysis 22, 366-389 (1976)

5. Bremer, I., Henrion, R., Möller, A.: Probabilistic constraints via SQP solver: Application to a renewable energy management problem. Computational Management Science 12, 435-459 (2015)

6. Calafiore, G.C., Campi, M.C.: The scenario approach to robust control design. IEEE Trans. Automat. Control 51, $742-753(2006)$

7. Daníelsson, J., Jorgensen, B., Samorodnitsky, G., Sarma, M., de Vries, C.G.: Fat tails, VaR and subadditivity. Journal of Econometrics 172, 283-291 (2013)

8. Dentcheva, D.: Optimisation models with probabilistic constraints. In: A. Shapiro, D. Dentcheva, A. Ruszczyński (eds.) Lectures on Stochastic Programming. Modeling and Theory, MPS-SIAM series on optimization, vol. 9, pp. 87-154. SIAM and MPS, Philadelphia (2009)

9. Dentcheva, D., Martinez, G.: Regularization methods for optimization problems with probabilistic constraints. Math. Programming (series A) 138(1-2), 223-251 (2013)

10. Dentcheva, D., Prékopa, A., Ruszczyński, A.: Concavity and efficient points for discrete distributions in stochastic programming. Mathematical Programming 89, 55-77 (2000)

11. Diniz, A.L., Henrion, R.: On probabilistic constraints with multivariate truncated gaussian and lognormal distributions. Energy Systems pp. 1-19 (2016)

12. Ermoliev, Y., Ermolieva, T., Macdonald, G., Norkin, V.: Stochastic optimization of insurance portfolios for managing exposure to catastrophic risk. Annals of Operations Research 99, 207-225 (2000)

13. Fang, K., Kotz, S., Ng, K.W.: Symmetric multivariate and related distributions, Monographs on Statistics and Applied Probability, vol. 36, 1st edn. Springer-Science (1990)

14. Farshbaf-Shaker, M.H., Henrion, R., Hömberg, D.: Properties of chance constraints in infinite dimensions with an application to pde constrained optimization. Set Valued and Variational Analysis pp. 1-21 (2017). DOI 10.1007/s11228017-0452-5

15. Garnier, J., Omrane, A., Rouchdy, Y.: Asymptotic formulas for the derivatives of probability functions and their Monte Carlo estimations. European Journal of Operations Research 198, 848-858 (2009). DOI 10.1016/j.ejor.2008.09.026

16. Geletu, A., Hoffmann, A., Klöppel, M., Li, P.: A tractable approximation of non-convex chance constrained optimization with non-gaussian uncertainties. Engineering Optimization 47(4), 495-520 (2015)

17. Hantoute, A., Henrion, R., Pérez-Aros, P.: Subdifferential characterization of continuous probability functions under gaussian distribution. Submitted preprint: https://arxiv.org/pdf/1705.10160.pdf pp. 1-27 (2017)

18. Henrion, R., Möller, A.: Optimization of a continuous distillation process under random inflow rate. Computer \& Mathematics with Applications 45, 247-262 (2003) 
19. Henrion, R., Möller, A.: A gradient formula for linear chance constraints under Gaussian distribution. Mathematics of Operations Research 37, 475-488 (2012). DOI 10.1287/moor.1120.0544

20. Henrion, R., Strugarek, C.: Convexity of chance constraints with independent random variables. Computational Optimization and Applications 41, 263-276 (2008)

21. Henrion, R., Strugarek, C.: Convexity of chance constraints with dependent random variables: the use of copulae. In: M. Bertocchi, G. Consigli, M. Dempster (eds.) Stochastic Optimization Methods in Finance and Energy: New Financial Products and Energy Market Strategies, International Series in Operations Research and Management Science, pp. 427439. Springer-Verlag New York (2011)

22. Hong, L., Yang, Y., Zhang, L.: Sequential convex approximations to joint chance constrained programed: A monte carlo approach. Operations Research 3(59), 617-630 (2011)

23. Kataoka, S.: A stochastic programming model. Econometrica 31, 181-196 (1963)

24. Kibzun, A., Uryas'ev, S.: Differentiability of probability function. Stoch. Anal. Appl. 16, 1101-1128 (1998). DOI $10.1080 / 07362999808809581$

25. Kogan, A., Lejeune, M.A.: Threshold boolean form for joint probabilistic constraints with random technology matrix. Mathematical Programming 147(1-2), 391-427 (2014)

26. Kogan, A., Lejeune, M.A., Luedtke, J.: Erratum to: Threshold boolean form for joint probabilistic constraints with random technology matrix. Mathematical Programming 155(1), 617-620 (2016)

27. Landsman, Z.M., Valdez, E.A.: Tail conditional expectations for elliptical distributions. North American Actuarial Journal 7(4), 55-71 (2013)

28. Lejeune, M., Margot, F.: Solving chance-constrained optimization problems with stochastic quadratic inequalities. Operations Research pp. 1-39 (2016)

29. Lejeune, M.A.: Pattern-based modeling and solution of probabilistically constrained optimization problems. Operations Research 60(6), 1356-1372 (2012)

30. Lejeune, M.A., Noyan, N.: Mathematical programming approaches for generating p-efficient points. European Journal of Operational Research 207(2), 590-600 (2010)

31. Lubin, M., Bienstock, D., Vielna, J.P.: Two-sided linear chance constraints and extensions. Arxiv 1507.01995 pp. 1-19 (2016)

32. Luedtke, J.: A branch-and-cut decomposition algorithm for solving chance-constrained mathematical programs with finite support. Mathematical Programming 146(1-2), 219-244 (2014)

33. Luedtke, J., Ahmed, S.: A sample approximation approach for optimization with probabilistic constraints. SIAM Journal on Optimization 19,674-699 (2008)

34. Marti, K.: Differentiation of probability functions : The transformation method. Computers and Mathematics with Applications 30, 361-382 (1995). DOI 10.1016/0898-1221(95)00113-1

35. Morgan, D., Eheart, J., Valocchi, A.: Aquifer remediation design under uncertainty using a new chance constraint programming technique. Water Resources Research 29, 551-561 (1993)

36. Naor, A., Romik, D.: Projecting the surface measure of the sphere of $\ell_{p}^{n}$. Ann. I.H. Poincaré 39(2), 241-261 (2003). DOI 10.1016/S0246-0203(02)00008-0

37. Nemirovski, A., Shapiro, A.: Convex approximations of chance constrained programs. SIAM Journal of Optimization 17(4), 969-996 (2006)

38. Pagnoncelli, B., Ahmed, S., Shapiro, A.: Sample average approximation method for chance constrained programming: Theory and applications. J. Optim. Theory Appl 142, 399-416 (2009)

39. Prékopa, A.: Programming under probabilistic constraints with a random technology matrix. Mathematische Operationsforchung und Statistik 5, 109-116 (1974)

40. Prékopa, A.: Stochastic Programming. Kluwer, Dordrecht (1995)

41. Prékopa, A.: On the concavity of multivariate probability distributions functions. Operations Research Letters 29, 1-4 (2001)

42. Prékopa, A.: Probabilistic programming. In: A. Ruszczyński, A. Shapiro (eds.) Stochastic Programming, Handbooks in Operations Research and Management Science, vol. 10, pp. 267-351. Elsevier, Amsterdam (2003)

43. Raik, E.: The differentiability in the parameter of the probability function and optimization of the probability function via the stochastic pseudogradient method (russian). Izvestiya Akad. Nayk Est. SSR, Phis. Math. 24(1), 3-6 (1975)

44. Rinott, Y.: On the convexity of measures. Annals of Probability 4, 1020-1026 (1976)

45. Royset, J., Polak, E.: Extensions of stochastic optimization results to problems with system failure probability functions. Journal of Optimization Theory and Applications 133(1), 1-18 (2007)

46. Shapiro, A., Dentcheva, D., Ruszczyński, A.: Lectures on Stochastic Programming. Modeling and Theory, MPS-SIAM series on optimization, vol. 9. SIAM and MPS, Philadelphia (2009)

47. Uryas'ev, S.: Derivatives of probability functions and integrals over sets given by inequalities. Journal of Computational and Applied Mathematics 56(1-2), 197-223 (1994). DOI 10.1016/0377-0427(94)90388-3

48. Uryas'ev, S.: Derivatives of probability functions and some applications. Annals of Operations Research 56, 287-311 (1995)

49. Uryas'ev, S.: Derivatives of probability and integral functions: General theory and examples. In: C.A. Floudas, P.M. Pardalos (eds.) Encyclopedia of Optimization, 2nd edn., pp. 658-663. Springer - Verlag (2009)

50. van Ackooij, W.: Eventual convexity of chance constrained feasible sets. Optimization (A Journal of Math. Programming and Operations Research) 64(5), 1263-1284 (2015). DOI 10.1080/02331934.2013.855211

51. van Ackooij, W.: Convexity statements for linear probability constraints with gaussian technology matrices and copulæ correlated rows. ResearchGate pp. 1-19 (2017). DOI 10.13140/RG.2.2.11723.69926 
52. van Ackooij, W., Berge, V., de Oliveira, W., Sagastizábal, C.: Probabilistic optimization via approximate p-efficient points and bundle methods. Computers \& Operations Research 77, 177-193 (2017). DOI 10.1016/j.cor.2016.08.002

53. van Ackooij, W., Frangioni, A., de Oliveira, W.: Inexact stabilized Benders' decomposition approaches: with application to chance-constrained problems with finite support. Computational Optimization And Applications 65(3), 637-669 (2016). DOI 10.1007/s10589-016-9851-z

54. van Ackooij, W., Henrion, R.: Gradient formulae for nonlinear probabilistic constraints with Gaussian and Gaussian-like distributions. SIAM Journal on Optimization 24(4), 1864-1889 (2014)

55. van Ackooij, W., Henrion, R.: (Sub-) Gradient formulae for probability functions of random inequality systems under Gaussian distribution. SIAM Journal on Uncertainty Quantification 5(1), 63-87 (2017)

56. van Ackooij, W., Henrion, R., Möller, A., Zorgati, R.: Joint chance constrained programming for hydro reservoir management. Optimization and Engineering 15, 509-531 (2014)

57. van Ackooij, W., Malick, J.: Second-order differentiability of probability functions. Optimization Letters 11(1), 179-194 (2017). DOI 10.1007/s11590-016-1015-7

58. van Ackooij, W., de Oliveira, W.: Level bundle methods for constrained convex optimization with various oracles. Computation Optimization and Applications 57(3), 555-597 (2014)

59. van Ackooij, W., de Oliveira, W.: Convexity and optimization with copulæ structured probabilistic constraints. Optimization: A Journal of Mathematical Programming and Operations Research 65(7), 1349-1376 (2016). DOI 10.1080/02331934.2016.1179302

60. van Ackooij, W., Sagastizábal, C.: Constrained bundle methods for upper inexact oracles with application to joint chance constrained energy problems. SIAM Journal on Optimization 24(2), 733-765 (2014)

61. Zadeh, Z.M., Khorram, E.: Convexity of chance constrained programming problems with respect to a new generalized concavity notion. Annals of Operations Research 196(1), 651-662 (2012) 